\title{
Resumen de actividades del Comité de Tuberculosis, Región Metropolitana, 2006. Selección de Temas
}

\author{
CARLOS PEÑA M.*
}

Activities of Tuberculosis Committee from Santiago, Chile, during 2006. A summary of selected sessions

Durante 2006, entre marzo y diciembre, se efectuaron 10 reuniones del Comité Metropolitano de Tuberculosis de la Región Metropolitana (RM), las que se realizan desde hace más de 30 años, dentro de las actividades del Programa Nacional de Control de la Tuberculosis (PCT).

Entre los temas presentados, han sido elegidos algunos de los más representativos.

Programa de evaluación de control de calidad de exámenes

Sra. Fabiola Arias Muñoz, Instituto de Salud Pública.

El Instituto de Salud Pública (ISP), efectúa gratuitamente a los Laboratorios de Tuberculosis del Sistema Público, el control de calidad de las baciloscopías y también de los medios de cultivo, lo que asegura un mayor nivel de confiabilidad de los laboratorios de la red.

Uno de los problemas clásicos es la contaminación de las muestras, pero ahora el tubo lleva una tapa rosca y prácticamente ya no se produce contaminación. Lo aceptable es menos de un $3 \%$ de contaminación según la calidad de la muestra y $1 \%$ por la manipulación, pero no debe superar más estos rangos.

Para la evaluación de calidad todas las discordancias son revisadas, lo que permite diferenciar si se debieron a un problema administrativo o un problema técnico.

Una vez detectado el problema, se capacita al personal en las áreas deficitarias y se sigue eva- luando cada mes para verificar las correcciones efectuadas.

Rutinariamente se revisan: 1) Las baciloscopías positivas de una cruz (+) o de pocos bacilos; 2) Las baciloscopías de más cruces, para verificar que estén bien informadas; 3) Un porcentaje de las baciloscopías negativas.

En el Laboratorio de Referencia del Instituto de Enfermedades Tropicales de Bruselas, Bélgica, se evalúa al ISP en los estudios de sensibilidad, garantizando su calidad. En la actividad relacionada a tuberculosis, el ISP dispone de 4 técnicos paramédicos, 2 auxiliares de servicio y 3 profesionales (falta un profesional).

\section{Resultados de la evaluación de baciloscopías de los servicios públicos:}

Se controlan habitualmente entre 750 y 890 láminas. En la última evaluación efectuada resultaron negativas 650 y positivas 240 . Los falsos (+) fueron $0,5 \%$ y los falsos (-) variaron entre 0,17 y $0,15 \%$. La discordancia total fue $0,17 \%$, lo que refleja la alta calidad del trabajo realizado en los laboratorios de la red pública.

\section{Resultados de la evaluación de baciloscopías de servicios privados: \\ Se evaluaron 1.076 láminas, resultando nega- tivas 993 y positivas 82 . Los falsos (+) fueron de $9,6 \%$ en el año 2003, lo que hizo necesario realizar una capacitación del personal. Luego de esta capacitación, en 2004 y 2005 no se presen- taron falsos (+), en tanto que los falsos (-) que se producían se redujeron de 0,42 a $0,1 \%$.}

\footnotetext{
* Coordinador Regional Metropolitano de Tuberculosis. Equipo Técnico de Tuberculosis. Servicio de Salud Metropolitano (SSM) Central.
} 
Estrategias de localización de casos de tuberculosis pulmonar

Dr. Álvaro Yáñez, Equipo Técnico de TBC. SSM Occidente y Dr. Fernando Tabilo, Equipo Técnico de TBC. SSM Sur-Oriente.

En el caso de la tuberculosis, toda la población beneficiaria debe ser atendida gratuitamente. Esta disposición continúa, sin ser alterada por las recientes reformas de la salud, de manera que a los directivos o jefes de los establecimientos locales, se les debería recordar, reiteradamente, la importancia de los programas de localización de casos, pesquisa o tamizaje de la tuberculosis. Tamizaje es una "estrategia pasiva", dirigida a los consultantes espontáneos de los Servicios de Salud, por la cual se debe buscar mucho para encontrar poco; sin embargo, así se diagnostican casos en los cuales nadie sospecha que exista una tuberculosis.

El tamizaje del sintomático respiratorio debe ser independiente de su motivo de consulta. Muchos enfermos no consultan directamente por sus síntomas respiratorios; los fumadores, por ejemplo, consideran normal tener "la tos del fumador" y, así, la demora en hacer el diagnóstico es de meses. En el $45 \%$ de los casos es de más de dos meses, y el $35 \%$ de éstos incluso de más de 6 meses. Los contactos de este paciente bacilífero, no diagnosticado oportunamente, están en riesgo de contagio por todo este tiempo. Es así como todos los estudios demuestran, que al momento del diagnóstico del caso índice, alrededor del $50 \%$ de sus contactos íntimos ya están infectados y 2 a 3\% de ellos ya está enfermo.

Sin embargo, en Chile se dejaron de efectuar $22 \%$ de las baciloscopías (BK) de pesquisa programadas el año 2005. En la región metropolitana se practicaron sólo $150.000 \mathrm{BK}$ ese año, o sea que hubo 30.000 BK menos que en 2004. Como por cada 200 baciloscopías practicadas, se diagnostica actualmente alrededor de un caso, esto significa que dejamos de diagnosticar 150 enfermos de TBC pulmonar, o sea que se nos estaría escapando una cuarta parte de las TBC pulmonares existentes.

En un estudio efectuado en el SSM Sur-Oriente ${ }^{1}$, el $50 \%$ de los bacilíferos diagnosticados ya estaba en control, dentro del sistema de salud por otras patologías y no era la primera vez que

${ }^{1}$ TABILO F, PEÑA C. Reorientación del programa de localización de casos de tuberculosis. Región Metropolitana, 2006. Rev Chil Enf Respir 2007; 23: 135-40. consultaba, cuando finalmente se les diagnosticó la TBC.

La población general no conoce la existencia de un Programa de Control de la Tuberculosis, porque no se ha publicitado por los medios masivos como la televisión, que es la que más llega a los usuarios. Tampoco se enseña a los estudiantes de medicina y de otras carreras relacionadas con la salud, que la morbi-mortalidad de la TBC, comparada con la de otras enfermedades infecto-contagiosas más de moda, como el Hanta, el SIDA o el SARS, es muy superior.

Actualmente, el diagnóstico se ha ido desplazando desde la atención primaria a la secundaria, de modo que muchos de los casos nuevos detectados proceden de los servicios de urgencias, de médicos particulares, de las "Campañas de Invierno" y de los especialistas.

\section{Requisitos para el tamizaje:}

- Debe existir una "decisión política destinada a poner en práctica esta estrategia”. Actualmente las baciloscopías de pesquisa están dentro de las "Orientaciones Programáticas", pero deberían estar más bien como "Compromisos de Gestión".

- Debe obtenerse la colaboración de los directores locales y de los jefes de los Departamentos de Salud Municipal y conseguir la participación de todos los médicos en las actividades de localización de casos de tuberculosis. Lamentablemente, no es evidente la presencia de los directores en las evaluaciones de los resultados de la pesquisa en el nivel local. Se sugiere incentivar la acción en los niveles locales y también en los directores del área ejecutiva y alcaldes. Los consultorios y comunas deben enviar sus resultados, como índice de pesquisa, cada mes, al nivel secundario.

- Se ha propuesto anotar en la ficha de cada enfermo, al lado de la fecha, un timbre como "Sintomático Respiratorio": "SR = Tiene examen de BK pedido". Otros sugieren estimular la solicitud de baciloscopías en la preconsulta, con el personal que trabaja en la ventanilla de admisión o con el/la técnico paramédico que registra los signos vitales, para que agregue la solicitud de baciloscopía, si el consultante es sintomático respiratorio.

- Debemos asociarnos a otros programas, como el de enfermedades cardiovasculares, de diabéticos, del adulto mayor, de pacientes postrados, etc.

- Debe mantenerse un continuo monitoreo y evaluación de las acciones. 
- Hay que lograr mayor participación del SAPU y de los servicios de urgencia.

- Debe disponerse de dinero para la educación y de transporte para concurrir a las supervisiones.

- Debe haber dinero, también para que los pacientes se movilicen a recibir el tratamiento supervisado (DOTS).

- Deben existir incentivos personales para el personal, no en dinero, sino como "notas de mérito", que finalmente, en forma indirecta, influirán en aumentar su sueldo.

- Debemos informar ampliamente que aunque la TBC está siendo controlada en Chile, esto se puede revertir si descuidamos las acciones del Programa de Control de la Tuberculosis (PCT), y hay que destacar que los pacientes no diagnosticados son peligrosos, porque contagian de una enfermedad potencialmente mortal.

- Debe hacerse pesquisa "activa" a los grupos de riesgo, según su localización geográfica y en poblaciones cerradas. Se debe conocer la distribución de la pobreza en cada territorio, porque es un importante factor asociado al riesgo de TBC y se deben evaluar las facilidades de acceso al sistema local de salud de cada comuna.

A pesar que la TBC no es considerada como una "enfermedad de urgencia", nuestra meta es que la población deje de ser infectada por el bacilo de Koch. Para cumplir con este objetivo hay que diagnosticar a los bacilíferos donde quiera que se encuentren.

\section{Comportamiento de la tuberculosis en la Región Metropolitana durante el 2005}

Dr. Carlos Peña, Equipo Técnico de TBC. SSM Central y Sra. Zulema Torres, EU, Sub-departamento Prioridades Sanitarias. Secretaría Regional Ministerial de Salud.

A pesar que actualmente es más difícil diagnosticar la enfermedad TBC, no se deben abandonar los esfuerzos diagnósticos a través de la baciloscopía (BK) de los sintomáticos respiratorios y el estudio de los contactos. Estos últimos permiten encontrar hasta un 2 a $3 \%$ de enfermos de TBC.

Los enfermos se concentran en áreas territoriales, aún dentro del mismo Santiago, con tasas tan disímiles que van de 8,8/100.000 a 60/ 100.000 , por lo que hay que concentrar la bús- queda en algunas unidades vecinales y comunales, en adultos mayores, indigentes, casas de reposo y reclusos.

\section{Evaluación de la TBC en la Región Metropolitana durante 2005}

Se identificaron un total de 967 enfermos tuberculosos, con una tasa de 15,3/100.000. La tendencia a la disminución de la incidencia es variable y oscila entre $-0,3$ y $-1,9 \%$, lo que está muy lejos de una reducción de $10 \%$ anual, que es lo que se requiere.

El 51,4\% de los casos son bacilíferos (497 casos, con una tasa de 7,8/100.000, mostrando una variación de $-0,9 \%$ con respecto al año previo).

Se presentaron tasas menores de 10/100.000 en los servicios: SSMOriente y SSMSur -Oriente; se identificaron 14 comunas de las 52 de la Región Metropolitana con tasas mayores de 20/ 100.000 .

La letalidad en la Región Metropolitana fue de $8,2 \%$. Las letalidades menores (bajo $5 \%$ ) ocurrieron en los servicios SSMNorte, SSMCentral y SSMOriente; las más altas, en SSMSur, SSMSur Oriente y SSMOccidente (entre 9,5 y $15,3 \%$ ). Se destaca la contribución de la población con factores de riesgo, que alcanza a un $35 \%$ de los casos. Los grupos de riesgo principales son los adultos mayores $(64 \%)$, VIH (+) $(14 \%)$, reos $(9 \%)$, indigentes $(4 \%)$ y extranje$\operatorname{ros}(9 \%)$.

\section{Evolución de la TBC en la Región Metropolitana entre 2001 y 2005}

La tasa de TBC en la Región Metropolitana se redujo de 17,4 a 15,1/100.000, pero los casos bacilíferos bajaron menos. La tasa en hombres es mayor que la de mujeres. El grupo de edad entre 15 y 64 años va disminuyendo en su incidencia. El grupo de TBC infantil es establemente bajo. El aumento de casos se presenta en el grupo de los adultos mayores.

Los indicadores que muestra cada servicio de salud se deben relacionar con las metas propuestas por la OMS y con los signos de alarma o de deterioro de la situación operacional, grado de reducción de la pesquisa, incremento de letalidad, reducción de las tasas de curación, reducción del cumplimiento del estudio de contactos, etc.

Se concluye que para cumplir las metas se deben asegurar:

1. Los compromisos de gestión. Que se dé cumplimiento a las estructuras organizativas, bajo responsabilidad del nivel central, Secretaría 
Regional Ministerial y de los servicios de salud, de modo que se cuente con todos los equipos de TBC completos, con horas asignadas a la gestión del PCT (evaluación, supervisión y capacitación).

2. Correcta aplicación de las Normas Técnicas del Programa.

\section{Daño hepático por drogas antituberculosas}

Dr. Carlos Peña M., Equipo Técnico de Tuberculosis. SSM Central.

Una de las reacciones adversas más temidas y que puede resultar en la muerte de un paciente, es la toxicidad hepática, por lo que es necesario estar alerta a la pesquisa precoz y a la oportuna suspensión del o los fármacos involucrados.

Se presentan los distintos mecanismos de daño hepático por drogas anti-TBC:

\section{Isoniazida (HIN)}

El HIN se acetila a hidrazina que es hepatotóxica. La producción de metabolitos aumenta cuando se dan otras drogas que estimulan al citocromo P450, como la rifampicina. Los acetiladores rápidos del HIN acumularían más rápido metabolitos tóxicos. El 50\% de las veces el daño se produce antes de los 2 meses de la administración del HIN.

Por adaptación a metabolitos tóxicos, el 10\% de las personas tiene elevación transitoria (de menos de un mes) de las enzimas hepáticas (transaminasa oxaloacética y pirúvica) a niveles plasmáticos inferiores a $200 \mathrm{UI} / \mathrm{L}$ y sólo un $0,5 \%$ hace una hepatitis clínica, que es más frecuente entre los mayores de 50 años de edad y en los portadores de daño hepático crónico (3\%). La letalidad de la hepatitis por HIN puede llegar al $10 \%$.

La toxicidad aumenta al asociar Rifampicina (R), Pirazinamida (Z), al consumir alcohol, al asociarse con hepatitis B, o cursar un embarazo o puerperio.

El riesgo de hepatitis durante la quimioprofilaxis con HIN es sólo de $0,1 \%$.

\section{Pirazinamida $(Z)$}

Cuando se asocia a HIN en dosis altas, mayores de $30 \mathrm{mg} / \mathrm{kg}$ al día, produce hasta un $15 \%$ de hepatitis. La hepatitis por $\mathrm{Z}$ suele ser de cierta gravedad, por lo que es riesgosa su indicación en pacientes con daño hepático previo.

\section{Rifampicina $(R)$}

Raramente produce daño hepático, incluso en alcohólicos y adultos mayores. Actualmente, en EE.UU., se está haciendo quimioprofilaxis con $\mathrm{R}$ en vez de HIN, lo que permite acortar la terapia preventiva a 4 meses.

Con $\mathrm{R}$ se puede presentar un patrón de ictericia de tipo colestásico, pero sin aumento de las enzimas hepáticas, porque hay competencia con la proteína transportadora relacionada con el metabolismo de la bilirrubina. Al suspender la R y reutilizarla luego de desaparecida la ictericia, ésta generalmente no se vuelve a producir. Se sugiere no sobrepasar, como dosis tope de R, los $900 \mathrm{mg} /$ día. La $\mathrm{R}$ puede potenciar la acción hepatotóxica del HIN al inducir más rápidamente la formación de metabolitos hepatotóxicos.

\section{Pacientes con riesgo de hepatotoxicidad a drogas antituberculosas}

1. Daño hepático previo.

2. $\mathrm{VIH}+$.

3. Embarazo y primeros 3 meses de puerperio.

4. Consumo habitual de alcohol.

5. Uso de otros fármacos hepatotóxicos.

\section{Cómo reconocer la hepatotoxicidad:}

Los síntomas son muy parecidos a la hepatitis viral, con anorexia, náuseas y vómitos. Puede existir ictericia, dolor abdominal, síndrome gripal, fiebre y mialgias. El control del nivel de enzimas hepáticas se debe realizar en los pacientes sintomáticos y en los de alto riesgo.

\section{Conducta ante la hepatotoxicidad:}

Ante la sospecha de daño hepático, por síntomas compatibles, hay que suspender todo el tratamiento. También es mandatorio suspenderlo si las enzimas hepáticas suben 5 a 6 veces sobre su valor de referencia normal, aún estando el paciente asintomático.

Luego, cuando las transaminasas se normalizan, se debe reiniciar la terapia, excluyendo el HIN y la Z que son las drogas que producen las hepatitis más frecuentes y graves. El esquema alternativo es: $2 \mathrm{SRE} / 10 \mathrm{R}_{2} \mathrm{E}_{2}$ (un año de tratamiento cuando se mantiene la $\mathrm{R}$ en ambas fases). La antigua norma decía que debería prolongarse a 18 meses cuando se mantiene el HIN en vez de la $\mathrm{R}$, pero actualmente se prefiere excluir el HIN.

Luego de iniciada la terapia alternativa se hacen pruebas hepáticas a la semana y al mes de tratamiento. 


\section{Consideraciones operacionales:}

- Daño hepático crónico no es lo mismo que insuficiencia hepática por drogas. No hay que iniciar un esquema de daño hepático en un consumidor de alcohol que no presenta insuficiencia hepática clínica o de laboratorio. Se debe siempre preferir incentivar al paciente a abandonar este hábito.

- El tratamiento supervisado (DOTS) permite el reconocimiento de las reacciones adversas a drogas (RAM), por lo que la administración controlada del tratamiento debe ser realizada por personal capacitado en el reconocimiento de las RAM. Nunca se deben entregar medicamentos para su autoadministración sin control del enfermo.

\section{Vulnerabilidad en salud: Un tema relacionado a tuberculosis}

Sra. Zulema Torres, EU y Dr. Patricio Olivares, Subdepartamento de Planificación y Prioridades Sanitarias. Secretaría Regional Ministerial de Salud.

El comportamiento de la TBC guarda relación con innumerables componentes sociales y biomédicos; basta recordar que se ha desarrollado incluso un puntaje de riesgo para predecir el comportamiento de los pacientes, como su adherencia al tratamiento o las probabilidades de éxito de la terapia. Estas variables son importantes de describir y analizar para el logro de los objetivos sanitarios. Estos objetivos para 2010 son:

1. Mejorar los logros ya alcanzados.

2. Enfrentar los desafíos del envejecimiento de la población.

3. Disminuir las desigualdades en salud o inequidad

4. Aumentar la participación y la calidad de prestación a los usuarios.

La TBC debe enmarcarse dentro de un plan de salud que es particular a la situación del lugar y al momento de su aplicación.

\section{Plan de salud pública regional}

La pobreza es el principal factor de riesgo "prevenible". Existe una correlación entre una mayor incidencia comunal de TBC y los indicadores de deterioro socioeconómico de dicha comuna.

En Chile, los pobres han disminuido de $971.000(16 \%)$ a $847.120(13,5 \%)$; siendo el
4,3\% de ellos indigentes. El alfabetismo es del $97 \%$, pero el $25 \%$ de la gente más pobre no tiene los 12 años de escolaridad. El analfabetismo cultural supera el $50 \%$. La población originaria corresponde a 3,3\% y de ellos el $41 \%$ está en la indigencia o en el quintil más pobre. Los discapacitados también están dentro del grupo de los más pobres.

En la Región Metropolitana, que al contar con 6.061 .850 habitantes, corresponde al $40 \%$ de los habitantes del país, el $96 \%$ de ellos es urbano. El 35\% de los enfermos tuberculosos de la Región Metropolitana tiene alguna condición o factor de riesgo. La mayoría de este riesgo está dado por los adultos mayores en cada servicio de salud: el $64 \%$ de los casos con factores de riesgo se deben a esta condición.

Conviene analizar los "mapas epidemiológicos", que permiten visualizar la concentración de riesgos poblacionales y compararlos en su evolución.

Se propone aplicar el Índice de Prioridad Social (IPS) por comuna. El IPS se basa en la evaluación de varios elementos, entre ellos los siguientes:

a) Educación o promedio de años de escolaridad.

b) Calidad de la vivienda.

c) Acceso a la salud, años de vida perdidos, tasa de fecundidad específica en mujeres menores de 19 años, embarazo en la adolescente (que en la Región Metropolitana ha aumentado en un $133 \%$ ).

Para dimensionar la pobreza se puede calcular el ingreso per cápita (suma de ingresos intrafamiliares relacionados con el número de personas de la familia), a fin de identificar a quienes no pueden costear su canasta básica mensual o los que ganan menos que el sueldo mínimo.

La indigencia es un grado mayor de pobreza y también está dimensionada (datos de personas en situación de calle, beneficiarios del Hogar de Cristo, y otros).

Se destaca la presencia de poblaciones con deterioro económico importante en Renca y Til Til, que requerirán de apoyo. Seguramente la TBC será un importante problema de salud en estas comunidades.

Se están recogiendo estadísticas a nivel de poblaciones penales y de extranjeros e inmigrantes, que son otros grupos donde la TBC es más prevalente. Los planes y diagnósticos comunales deben considerar estos componentes, actualizarlos y apoyarse sinérgicamente en 
las dos Subsecretarías actuales, la de Salud Pública que diseña la planificación y la de Redes Asistenciales, que ejecuta la actividad.

\section{Discusión}

El Dr. Álvaro Yáñez comenta que la TBC sigue siendo un problema de Salud Pública a pesar que:

1. Existe una base epidemiológica de intervención.

2. Hay tecnología de calidad para el diagnóstico y el tratamiento.

3. Existen protocolos de lo que corresponde hacer.

El adulto mayor es uno de los mayores grupos de riesgo para la TBC. Cuando el adulto mayor era un infante, se infectó en un alto porcentaje con el bacilo tuberculoso y tiene el riesgo de desarrollar la enfermedad, cuando su sistema inmune sufra un deterioro. La proporción de enfermos adultos mayores alcanza actualmente a un $8-11 \%$ de los casos de TBC en la población general.

Dado que la población de mayor edad fue infectada en un alto porcentaje y puede reactivar la enfermedad, la meta a lograr es que sólo existan estallidos ocasionales en estos grupos de mayor riesgo.

Las actividades de extensión del programa ya se están efectuando, por ejemplo en Gendarmería se capacitó un equipo técnico, que funciona como un consultorio más y envía información mensualmente.

Se propone que al ingreso a la cárcel se pida baciloscopía a todos los sintomáticos respiratorios. Aunque Gendarmería depende del Ministerio de Justicia y no del Ministerio de Salud, estas unidades han respondido adoptando las estrategias y actividades del PCT ministerial, contenidos en el Manual de Normas Técnicas vigentes.

Con respecto a los otros grupos de riesgo, se propone intervenir suministrando información a clubes de adultos mayores y a la comunidad, con la formación de monitores, entre los cuales se puede contar con algunos ex pacientes motivados. También es necesario integrarse con las actividades de otros programas como postrados, cardiovasculares y diabéticos, ya que debemos recordar que el $50 \%$ de los bacilíferos diagnosticados entre los sintomáticos respiratorios, se encontraba en control de crónicos al momento del diagnóstico.

Otra de las actividades a corto plazo que se proponen sería calcular las tasas específicas para los distintos grupos de riesgo, repetir y ampliar los estudios de "demora diagnóstica" y crear un comité en la Región Metropolitana, que se ocupe de la vigilancia de los distintos grupos de riesgo. 